\title{
Effects of biopsychosocial factors on the association between loneliness and mental health risks during the COVID-19 lockdown
}

\author{
Olga Megalakaki ${ }^{1,2} \cdot$ Cyrille Kossigan Kokou-Kpolou $^{3}$ (D)
}

Accepted: 17 August 2021 / Published online: 21 August 2021

(C) The Author(s), under exclusive licence to Springer Science+Business Media, LLC, part of Springer Nature 2021

\begin{abstract}
Previous data suggest that loneliness is a hallmark of the mental health issues triggered by the SARS-CoV-2 pandemic. The purpose of the present study was to examine the impact of loneliness on mental health and behavioural outcomes, as well as the moderating and mediating effects of biopsychosocial variables on these relationships. The data were collected during France's first national COVID-19 lockdown and included 556 participants $\left(M_{\text {age }}=30.06\right.$ years, range $\left.=18-87\right)$ who completed well-known validated measures assessing symptom-levels of anxiety, depression, and insomnia. They also indicated their level of worry about the COVID-19 crisis, and provided sociodemographic and health status data. Responses were submitted to hierarchical linear regression and mediation analyses. In terms of prevalence of loneliness, $18.9 \%$ of participants reported severe loneliness. High levels of loneliness were significantly associated with the three mental health and behavioural outcomes. The loneliness - anxiety relationship was moderated by employment and working arrangements. The loneliness - insomnia relationship was moderated by living conditions, history of medical or psychological problems, and COVID-19-related worry. Further findings supported the indirect effects of COVID-related worry on pathways from loneliness to the three mental health and behavioural health outcomes. The mediation models accounted for $28.9 \%, 33.7 \%$, and $15.0 \%$ of the variance in anxiety, depression, and insomnia. The present evidence-based findings revealed that loneliness and worry were major contributing factors of mental health and behavioural concerns during the COVID-19 lockdown. They could inform treatment recommendations for tackling prolonged-self isolation and loneliness in specific vulnerable groups.
\end{abstract}

Keywords Loneliness $\cdot$ Anxiety $\cdot$ Depression $\cdot$ Insomnia $\cdot$ Worry $\cdot$ Mediators $\cdot$ Moderators $\cdot$ COVID-19

\section{Introduction}

On 24 January 2020, the first official COVID-19 cases were announced in France. By January 2021, France had become the third most affected European country in terms of COVID-19 cases and deaths. In response to successive outbreaks, the French Government has imposed strict public health measures, including a total national lockdown during the first outbreak, when

Cyrille Kossigan Kokou-Kpolou

kkokoukp@uottawa.ca

1 Centre de Recherche en Psychologie: Cognition, Psychisme, Organisations (CRP-CPO), University of Picardy Jules Verne, Amiens, France

2 Sigmund Freud University, Paris, France

3 School of Psychology (Clinical), University of Ottawa, 136 Jean-Jacques-Lussier 4085, Vanier Hall, Ottawa, Ontario K1N 6N5, Canada universities, and nonessential businesses had to close, and sports, religious and cultural events were cancelled. In addition to public health measures, social distancing has become the main policy in terms of public behaviour. Research has shown that even though public health measures like these are necessary to curb the rapid worldwide spread of the virus, they have affected the psychological wellbeing and mental health of large portions of the world's populations (Brooks et al., 2020). Meta-analytic findings have revealed high prevalence rates of severe mental health conditions, including posttraumatic stress disorder, anxiety, depressive symptoms, and sleep problems among COVID-19 survivors, healthcare workers, and the general public (Cénat et al., 2021; Marvaldi et al., 2021). The unknowns and uncertainties surrounding the timeline of the pandemic are additional sources of overwhelming stress and worry (Paluszek et al., 2021; Peng et al., 2021). Furthermore, the immediate COVID-19-induced stress during the first outbreak was found to predict subsequent development of mental health and behavioural risks (Megalakaki et al., 2021). 


\section{Loneliness and Mental Health Issues COVID-19 Pandemic}

Researchers have argued that the subjective loneliness resulting from social distancing and stay-at- or working-from-home arrangements is a hallmark of the mental health problems caused by the SARS-CoV-2 pandemic (Jeste et al., 2020; Saltzman et al., 2020). Although some studies have reported stable levels of loneliness (Bu et al., 2020a; Luchetti et al., 2020), most available data show statistically significant increases in loneliness levels during national lockdowns and compared with prepandemic levels (Killgore et al., 2020a, 2020b, 2020c; McGinty et al., 2020).

Over the years, associations between loneliness and physical and mental health problems, as well as impaired cognitive functioning and mortality risks, have been extensively documented (Beutel et al., 2017; Hawkley \& Cacioppo, 2010; Holt-Lunstad et al., 2015; Lara et al., 2020; Leigh-Hunt et al., 2017; Lim et al., 2020; Solmi et al., 2020). With a few exceptions, studies of individuals across the lifespan have shown that loneliness is particularly prevalent among females, younger and elderly people (U-shaped curve), the jobless, single people, and people with mental and chronic physical health conditions (Hawkley \& Cacioppo, 2010; Solmi et al., 2020).

In the context of the COVID-19 crisis, research indicates that greater loneliness is positively associated with greater anxiety and depressive symptoms (Killgore et al., 2020a; Okruszek et al., 2020; Palgi et al., 2020; Tso \& Park, 2020), suicidal ideation (Killgore et al., 2020a), sleep problems (Grossman et al., 2021; Kokou-Kpolou et al., 2020a), and signs of psychosis risk (Tso \& Park, 2020). In adult samples in the United Kingdom, two studies (Bu et al., 2020b; Groarke et al., 2020) found high loneliness levels in young people, people on lower incomes, the economically inactive, and people with mental health conditions. Furthermore, Groarke et al. (2020) reported that living with others or in a rural area, and having more close friends or greater social support were protective factors. Findings for sex-related differences are mixed (Bu et al., 2020b; Groarke et al., 2020; Wickens et al., 2021). In a German adult sample, Buecker et al. (2020) found that daily loneliness increased more strongly for parents than for people without children. However, these studies did not examine the interactions between loneliness and these group factors in relation to mental health problems. A study conducted in Israel by Grossman et al. (2021) reported that sleep problems are associated with the loneliness-worry interaction, with lonely individuals with higher levels of worry about the pandemic reporting severe sleep problems.

\section{Worry about COVID-19 Crisis and Mental Health Problems}

A number of studies have established that worry about COVID-19, including fear about the future and fear of contracting the virus, as well as economic and health concerns, is associated with poor mental health and behavioural problems (Kämpfen et al., 2020). Worry can be conceptualized as a negatively valenced perseverative cognition or repetitive thoughts focused on sources of potential threat and oriented toward the future (Borkovec et al., 1983). According to these authors, "The worry process represents an attempt to engage in mental problem-solving on an issue whose outcome is uncertain but contains the possibility of one or more negative outcomes" (Borkovec et al., 1983, p. 10).

As process that cuts across specific clinical syndromes (Kircanski et al., 2015), worry is often associated with health problems (Brosschot et al., 2006; Clancy et al., 2020). Recent research suggests that perseverative cognition (i.e., worry and rumination) moderates the stress - sleep relationship (Tousignant et al., 2019). A longitudinal study revealed that metacognitive beliefs and worry mediate the relationship between different forms of loneliness (i.e., in family, romantic and social relationships) and both anxiety and depressive symptoms (Anyan et al., 2020). Recent evidence also suggests that worry about the COVID crisis mediates the association between perceived vulnerability to COVID-19 and posttraumatic stress (Boyraz et al., 2020). Consistent with these emerging data, we hypothesized that in addition to its moderating effect, worry about the COVID-19 crisis mediates the pathways from loneliness to mental health and behavioural problems.

\section{Overview of the Present Study and Research Questions}

Taken together, the above-mentioned studies suggest that loneliness in the unfolding pandemic situation is associated with a variety of psychopathologies, but not necessarily in a uniform way across different sociodemographic groups. However, there is still a clear lack of empirical knowledge about the interactions between loneliness and these group factors in relation to mental health problems in the current COVID-19 literature, and closing this lacuna would allow the development of evidence-based health interventions for specific vulnerable groups. Researchers have made some recommendations for tackling prolonged self-isolation and loneliness during the SARS-CoV-2 pandemic, based on previous studies conducted in a nonepidemic context (Beam \& Kim, 2020). However, it is still unclear just how relevant these recommendations are for vulnerable individuals with mental health concerns induced by the pandemic. In addition, emerging data suggest that loneliness has a differential impact on adverse health outcomes in different age groups, with changes in loneliness during the pandemic accounting for much of the increase in depressive symptoms - but not anxiety - among young adults (Bu et al., 2020b; Lee et al., 2020). Therefore, it is highly worthwhile using multiple mental health outcome assessments to gain an in-depth view of potential differences 
in the health consequences of loneliness across different sociodemographic groups during the pandemic.

To address these issues, we used data collected during the first nationwide lockdown in France to examine the associations between loneliness and three mental health and behavioural outcomes, namely anxiety, depressive symptoms, and insomnia. Of utmost interest, we investigated biopsychosocial factors that might moderate or mediate these associations. Based on previous studies (Bu et al., 2020a; Buecker et al., 2020; Groarke et al., 2020; Grossman et al., 2021; Hawkley et al., 2020), we considered 10 potential biopsychosocial factors: sex, age, place of residence, working arrangements, living conditions, marital status, presence of children, preexisting physical or mental health conditions, and COVID-related worry. Three primary research questions were addressed:

1. Is loneliness associated with anxiety, depressive symptoms, and insomnia?

2. Is there any evidence that the relationships between loneliness and the three mental health and behavioural outcomes are moderated by the selected biopsychosocial factors?

3. Is there any evidence that these relationships are mediated by worry about the COVID-19 crisis?

\section{Materials and Methods}

\section{Data Collection and Ethics}

To answer the primary research questions, this study used the cross-sectional and observational data which were collected in France between three and 16 May 2020. The details on the data source and methodology can be found in the previous article by Kokou-Kpolou et al. (2020b) which aimed at examining the prevalence and correlates of insomnia. To administer a survey through SurveyMonkey®, a secure online survey platform, the study used a nonprobability sampling strategy. Potential participants aged 18 years and above were invited to take part via social media, online advertising, community websites, and students' online newsletters. The study protocol adhered to the guidelines of the Declaration of Helsinki and its later revisions, and was approved by the Ethics Committee of the Faculty of Psychotherapy and the Faculty of Psychology, $\mathrm{S}$ i g m und Fre u d University-Paris (LBP2CFYKAO1BEX87902). Accordingly, after being informed about the purpose and nature of the study, participants gave their electronic informed consent as a condition for taking part. They were also informed about their right to withdraw from the study at any time without any justification. They all participated on a voluntary basis and did not receive any financial compensation.
Given the unknown targeted population size and the convenience sampling method used, the minimum required sample was set at 384 participants (Cochran, 1977). However, following the recommendation by Cohen et al. (2014), we expected a minimum sample size of 403 for computing power for regression coefficients and detecting the mediated effects (Fritz \& MacKinnon, 2007).

\section{Participants}

A total of 559 participants registered to take part, but three were excluded owing to spurious data (i.e., straight-line responses to closed measures). The final sample therefore comprised 556 adults $\left(75.5 \%\right.$ female; $M_{\text {age }}=30.06$ years, $S D=$ 14.38 ) and was considered adequate for the present analyses. The majority of participants $(88.7 \%)$ had a university education. The sample was composed of $52 \%$ university students, $38.1 \%$ full- or parttime employees, and $9.9 \%$ unemployed or retired. Regarding marital status, $46.2 \%$ were single, $50.2 \%$ were married or in a couple, and $3.6 \%$ were divorced or widowed, while $78.1 \%$ had no children, and $21.9 \%$ had one or more children. Nearly $17 \%$ reported medical or psychological antecedents. The majority of the participants (80\%) lived in an urban environment (town of more than 10,000 inhabitants). See Table 1 for detail of participants characteristics.

\section{Assessment Instruments}

\section{Outcome Measures}

Anxiety and Depressive Symptoms We used the Generalized Anxiety Disorder-7 (GAD-7; Spitzer et al., 2006) and the Patient Health Questionnaire (PHQ-9; Kroeke \& Spitzer, 2002) to measure anxiety and depressive symptoms experienced over the previous 2 weeks. In both measures, each item was rated on a 4-point Likert scale ranging from 0 (Not at all) to 3 (Almost every day). Possible scores ranged from 0 to 21 for GAD-7, and 0 to 27 for PHQ-9, with higher scores indicating more severe anxiety or depressive symptoms. In the present study, GAD-7 and PHQ-9 both had good internal reliability ( $\alpha=0.81$ and $\alpha=0.85$ ).

Insomnia: We used the Insomnia Severity Index (ISI) a 7-item self-report questionnaire, to assess seven aspects of insomnia (Morin et al., 2011). These included sleep onset, sleep maintenance, early morning awakening, interference with daily functioning, perceived prominence of impairment attributed to the sleep problem, concerns about sleep problems, and satisfaction with sleep patterns. Perceived severity of each item was rated on a $0-4$ scale. The seven ratings were summed to obtain a total score ranging from 0 to 28. Previous studies had reported good psychometric properties for both the English- and French-language versions (Bastien, 2001; Blais et al., 1997). 
Table 1 Characteristics of the study sample

\begin{tabular}{|c|c|c|}
\hline \multirow{3}{*}{ Age. M(SD) } & \multicolumn{2}{|c|}{ Sample $(N=556)$} \\
\hline & $\mathrm{n}$ & $\%$ \\
\hline & \multicolumn{2}{|c|}{$30.06(14.38)$} \\
\hline \multicolumn{3}{|l|}{ Gender } \\
\hline Male & 136 & 24.5 \\
\hline Female & 420 & 75.5 \\
\hline \multicolumn{3}{|l|}{ Marital status } \\
\hline Single & 257 & 46.2 \\
\hline In relationship & 108 & 19.4 \\
\hline Married & 171 & 30.8 \\
\hline Separated/divorced/widowed & 20 & 3.6 \\
\hline \multicolumn{3}{|l|}{ Working arrangements } \\
\hline Work at service & 73 & 13.1 \\
\hline Work from home & 289 & 52.0 \\
\hline At service and from home & 111 & 20.0 \\
\hline Loss of job & 17 & 3.1 \\
\hline \multicolumn{3}{|l|}{ Number of children } \\
\hline 0 & 434 & 78.1 \\
\hline 1 and more & 122 & 21.9 \\
\hline \multicolumn{3}{|c|}{ Living conditions during Covid-19 } \\
\hline Alone & 103 & 18.5 \\
\hline With family or other relatives & 453 & 81.5 \\
\hline \multicolumn{3}{|l|}{ Residence area } \\
\hline Capital & 99 & 17.8 \\
\hline Big town & 255 & 45.9 \\
\hline Small town & 91 & 16.4 \\
\hline Rural area & 111 & 20.0 \\
\hline \multicolumn{3}{|c|}{ Pre-existing mental/health problems } \\
\hline No & 463 & 83.3 \\
\hline Yes & 93 & 16.7 \\
\hline
\end{tabular}

\section{Predictors and Moderators}

Loneliness Loneliness was assessed using the UCLA 3-Item Loneliness Scale (Hughes et al., 2004). This scale measures three different aspects of loneliness: social connectedness, relational connectedness, and self-perceived connectedness. Each item is rated on a 3-point scale: 1 (Hardly ever), (Some of the time), and 3 (Often). Higher summed scores indicate greater degrees of loneliness. The cut-off point for loneliness was 6 , in line with previous studies. The scale exhibited acceptable internal reliability in our sample $(\alpha=0.71)$.

COVID-19 Related Worry This was assessed with a single item, rated on a scale ranging from 1 (Not at all) to 5 (Extremely). Participants were asked to report their level of concern about the COVID-19 crisis.
Sociodemographics and Health Status Participants provided information on their age, sex, education level, employment and working arrangements, living conditions, marital status, number of children, and place of residence. Data on preexisting mental and physical health conditions were also collected via a dichotomous question.

\section{Data Analysis}

Data were analysed using Mplus v. 8 (Muthen \& Muthen, 2017). Preliminary analyses consisted in checking whether the timing of our data collection influenced the assessment of our primary variables (i.e., loneliness, anxiety, depressive symptoms, and insomnia). The first national lockdown in France lasted from 17 March to 11 May 2020. We therefore verified whether participants who responded to the survey before the end of lockdown was announced $(n=462)$ differed from those who responded after the announcement $(n=$ $94)$ on any primary variables. As no significant differences were observed $(p \geq 0.22)$, we pooled the data of these two subgroups.

Following this step, we carried out descriptive statistics (frequency, mean, standard deviation) to summarize the data. The assumption of normal distribution of the data was also verified via skewness and kurtosis tests. No violations were observed, as all skewness values ranged between 0.58 and 1.27 , and all and kurtosis values ranged between -0.27 and 0.96 . Next, Pearson product-moment correlations were calculated out to verify the associations (and the directionality of these associations) of the main continuous variables. We then ran a series of moderation analyses through hierarchical linear regression models. Loneliness scores were entered in the model in the first step, followed by a multiplicative term representing the interaction between loneliness and each selected moderator in the second step, while controlling for other covariates in the third step. To reduce occurrence of multicollinearity among variables and aid in the interpretation of interactions, continuous variables were mean centered. The level of significance was set at .05 ; however, the marginally significant statistics for interactive effects were also considered (Kennedy-Shaffer, 2019).

Finally, we performed three mediation analyses considering loneliness as a predictor variable, anxiety, depression and insomnia as outcome variables, respectively, and COVID-19 related worry as a potential mediator. The indirect effects were examined using bootstrapping methods, as recommended by Preacher and Hayes (2008). We used 5000 bootstrap resamples from the original dataset $(N=556)$ to calculate the bias-corrected and accelerated $95 \%$ confidence interval $(95 \% \mathrm{BCa}$ CI). If the $\mathrm{CI}$ of the outcome of the mediation effect did not contain zero, then the mediation effect would be significant at the .05 level. 


\section{Results}

\section{Descriptive and Correlational Analyses}

In our sample, the mean score on the UCLA Loneliness scale was $4.31(S D=1.60)$, and $18.9 \%(95 \%$ CI $[15.7,22.4])$ of participants reported severe loneliness during the first COVID-19 lockdown in France. As can be seen in Table 2, Pearson product moment correlations showed that higher levels of loneliness correlated significantly with higher levels of COVID-19-related worry $(r=.18)$, greater anxiety $(r=$ $.42)$, more depressive symptoms $(r=.46)$, and greater insomnia $(r=.27)$, all $p$ s $<.001$.

\section{Tests for Moderation Models}

In all the hierarchical linear regression models, after controlling for covariates, high loneliness scores were still significantly associated with anxiety and depressive symptoms. See Supplementary Material for Tables (1-4). High loneliness scores were also significantly associated with insomnia, except for models where we entered the multiplicative terms Loneliness * Place of residence and Loneliness * Marital status. In total, we found four significant interactions: one between loneliness and anxiety, and three between loneliness and insomnia. First, working arrangements were found to moderate the relationship between loneliness and anxiety ( $B$ $=1.75, S E=0.57, p<.001)$. As depicted in Fig. 1 , when we plotted this interaction, we found that while higher levels of loneliness were associated with greater anxiety, this association was stronger among individuals who had lost their job because of the pandemic. Of note, the Loneliness * Working arrangements interaction almost significantly predicting insomnia symptoms, such that feelings of loneliness among individuals who worked from home $(\beta=-0.87, S E=0.50, p$ $=.08)$, followed by those who still went out to work ( $\beta=$ $-0.71, S E=0.38, p=.06$ ), tended to be associated with greater insomnia. Second, living conditions moderated the loneliness - insomnia relationship $(\beta=-1.02, S E=0.37, p$ $<.001)$. Visual inspection of Fig. 2 showed that participants with higher loneliness scores and who lived without family or other close relatives reported more severe insomnia. Third, the association between loneliness and insomnia varied according to health antecedents $(B=-0.80, S E=0.40, p<.01)$. As can be seen in Fig. 3, individuals with preexisting health problems who experienced greater feelings of loneliness also had an increased likelihood of reporting insomnia. Fourthly, loneliness and COVID-19-related worry interacted with each other in predicting insomnia $(B=0.30, S E=0.13, p<.05)$. More specifically, the loneliness - insomnia relationship was stronger among individuals with higher levels of COVID-19-related worry than those who reported lower levels of COVID-19-related worry (see Fig. 4).

\section{Tests for Mediation Models}

Figures 5, 6, and 7 summarize the results of the bootstrapped regression and mediation models for the effects of loneliness on anxiety, depressive symptoms, and insomnia through COVID-19-related worry, after controlling for all covariates. All three models were significant, and accounted for $28.9 \%$, $33.7 \%$, and $15.0 \%$ of the variance in anxiety, depressive symptoms, and insomnia. Loneliness was significantly associated with COVID-19 related worry $(B=0.09, S E=.03, p$ $<.001)$, and had direct effects on anxiety $(B=1.20, S E=$ $.05, p<.001)$, depressive symptoms $(B=1.37, S E=.13, p$ $<.001)$, and insomnia $(B=0.88, S E=.10, p<.001)$. The 95\% BCa CI for the mediated effects of COVID-19-related worry for all models did not contain 0 , suggesting that COVID-19 related worry was a significant mediator in the relationship between loneliness and the three mental health and behavioural outcomes. Moreover, for all the mediation paths we tested, results revealed that the direct effects of COVID-19 related worry on the three outcomes remained significant, suggesting partial mediation.

\section{Discussion}

\section{Main Findings of the Present Study}

The overarching aim of this study was to examine the impact of loneliness on anxiety, depressive symptoms and insomnia, and to explore whether some selected biopsychosocial
Table 2 Means, standard deviations (SD), range, and Pearson product moment correlations between the primary variables

\begin{tabular}{lcccccccc}
\hline & Mean & SD & Range & 1. & 2. & 3. & 4. & 5. \\
\hline 1. UCLA scores & 4.31 & 1.60 & {$[2-9]$} & 1.00 & & & \\
2. Covid-19-related worry & 3.18 & 1.08 & {$[1-5]$} & $.183^{* *}$ & 1.00 & & \\
3. GAD-7 scores & 7.16 & 5.25 & {$[0-27]$} & $.274^{* *}$ & $.184^{* *}$ & 1.00 &. & \\
4. PHG-9 scores & 9.02 & 5.66 & {$[0-28]$} & $.458^{* *}$ & $.191^{* *}$ & $653^{* *}$ & 1.00 & \\
5. ISI scores & 9.02 & 5.66 & {$[0-28]$} & $.274^{* *}$ & $.184^{* *}$ & $.940^{* *}$ & $.653^{* *}$ & 1.00 \\
\hline
\end{tabular}

**. All correlation coefficients are significant at the 0.01 level (2-tailed) 
Fig. 1 Moderating effect of resilience on the relationship between loneliness and anxiety. Note: Employment and working arrangements were recoded into « loss of job » and « other »

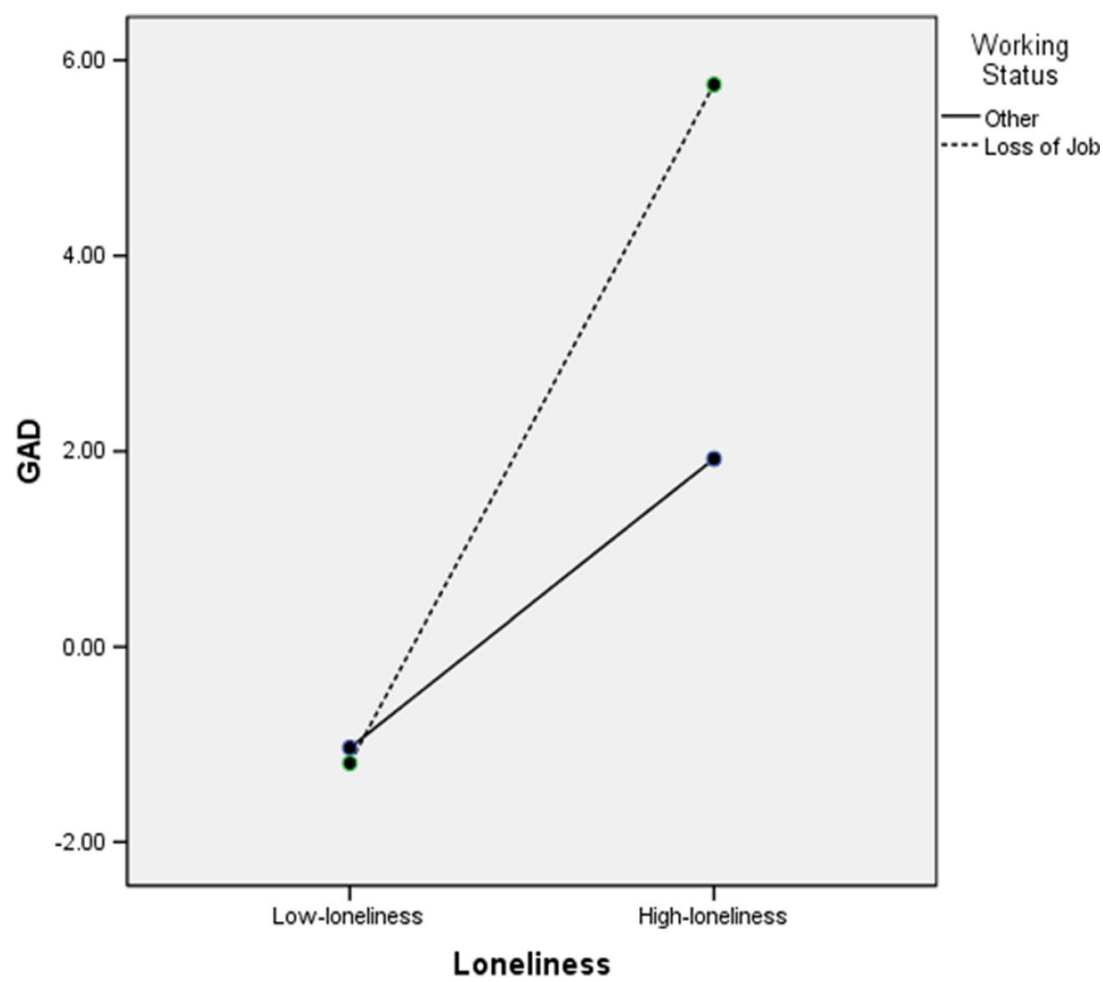

variables moderated or mediated these relationships. Taken together, findings indicated that greater loneliness was associated with greater anxiety, depressive symptoms, and insomnia in our sample during the first COVID-19 lockdown in France. In almost all the hierarchical linear regression models, these relationships remained statistically significant after accounting for covariates. This indicates that some sociodemographic factors, preexisting health status and COVID-19-related worry may contribute to anxiety, depressive symptoms, and insomnia. Across all regression models, employment and working arrangements modified the relationship between loneliness and anxiety. Furthermore, living conditions, history of medical or psychological conditions, and COVID-19-related worry moderated the relationship
Fig. 2 Moderating effect of living conditions on the relationship between loneliness and insomnia

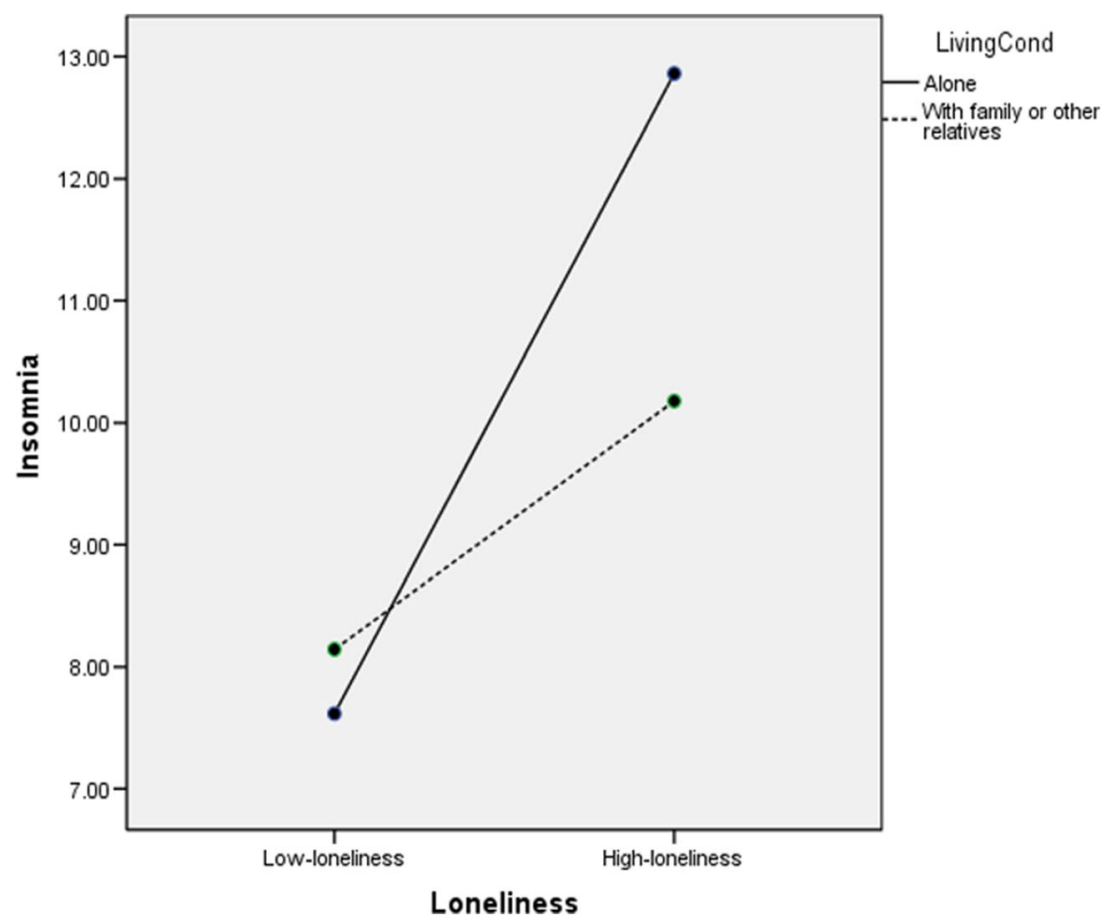


Fig. 3 Moderating effect of preexisting health problems on the relationship between loneliness and insomnia

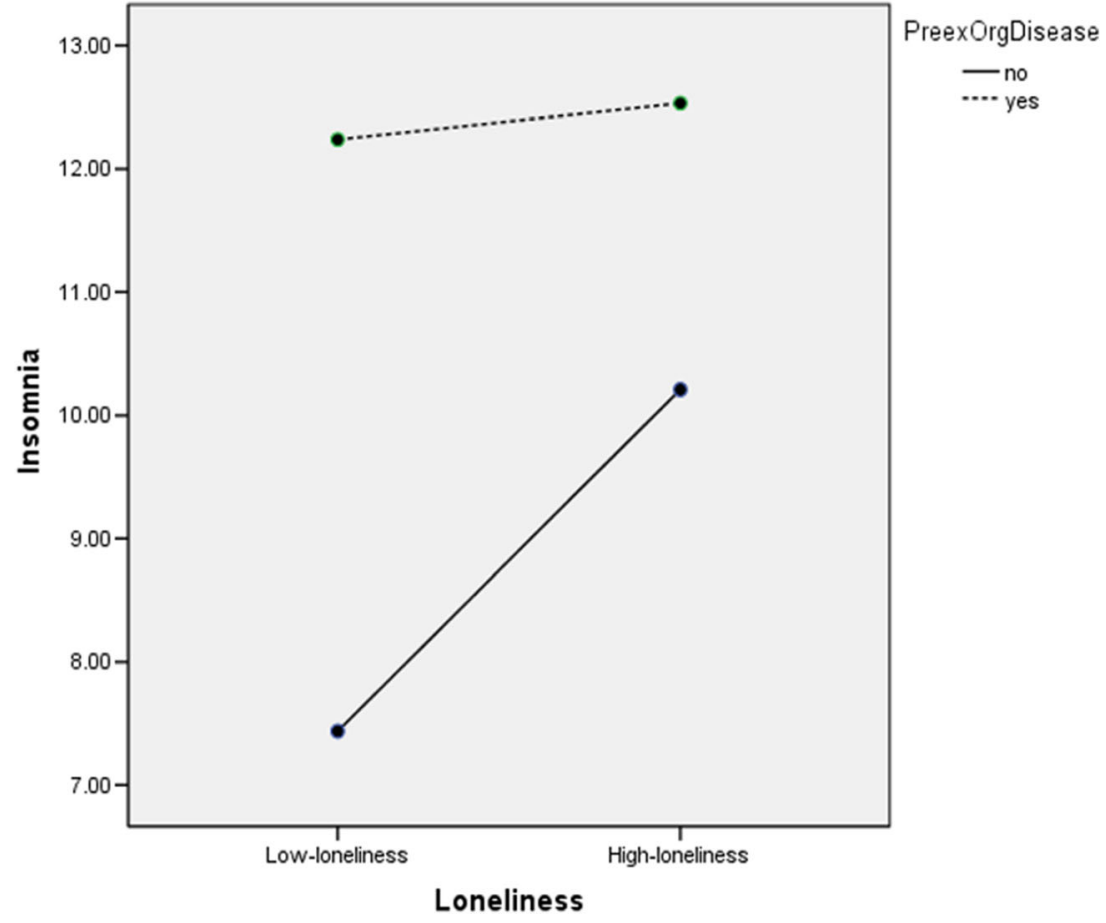

between loneliness and insomnia. No such evidence of moderating effects emerged for the relationship between loneliness and depressive symptoms. Furthermore, consistent with the third research question, we found evidence of the mediating effects of CoVID-related worry on pathways from loneliness to anxiety, depressive symptoms, and insomnia.

\section{Comparison with Other Studies and Explanation of Findings}

The weighted prevalence of severe loneliness was nearly $19 \%$ in our sample, which is similar to that reported in American (McGinty et al., 2020; Rosenberg et al., 2021) and Dutch (van der Velden et al., 2020, 2021) adult samples, but
Fig. 4 Moderating effect of Covid-19 related worry on the relationship between loneliness and insomnia

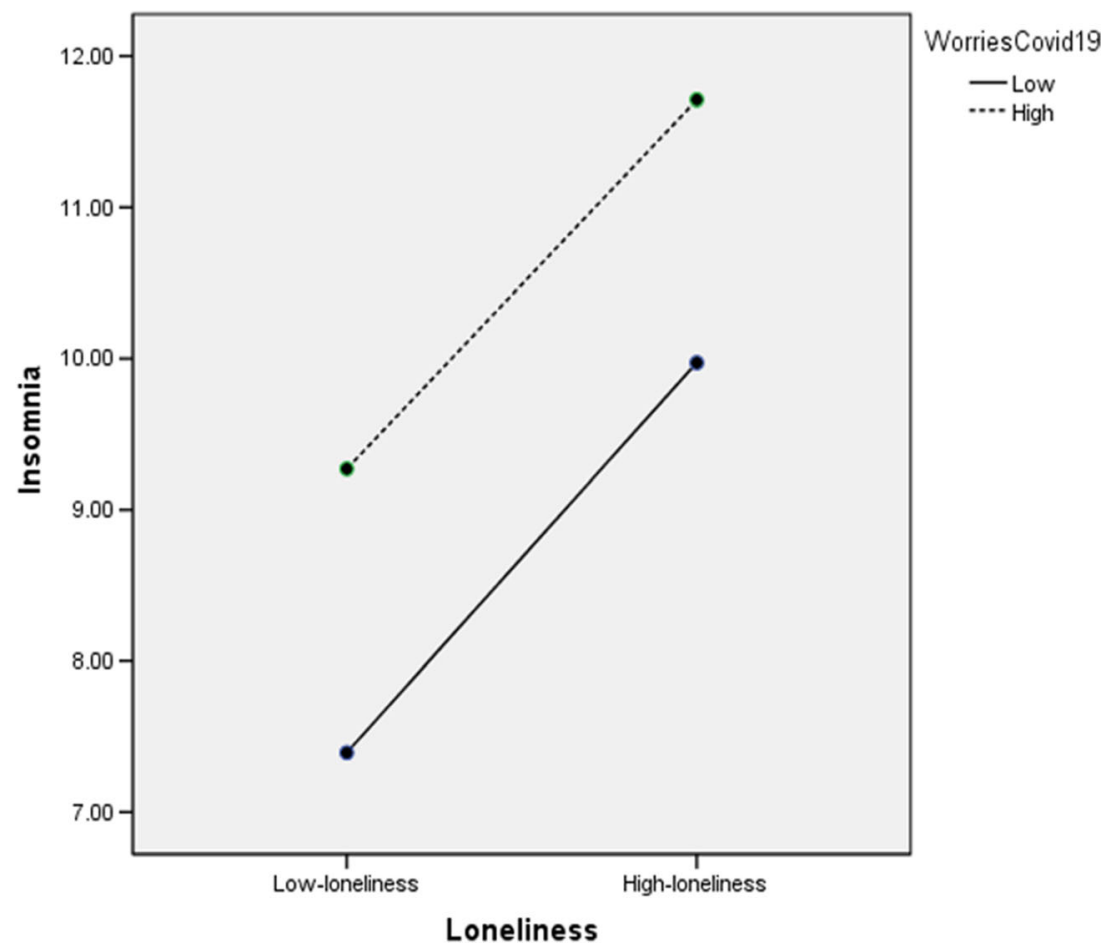




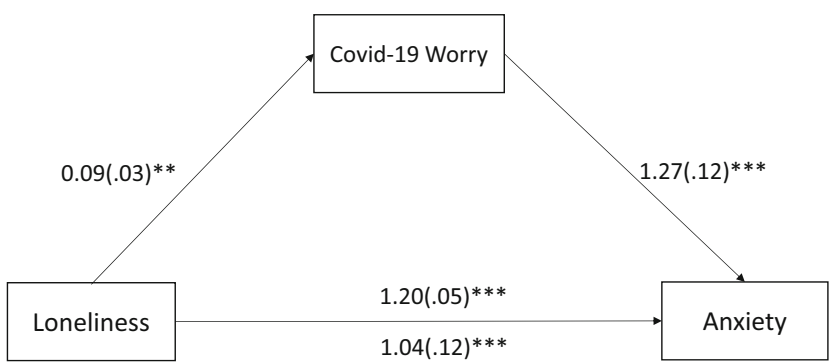

Fig. 5 Hypothesized path model showing mediated relationships between loneliness and anxiety through Covid-19 related worry. Rsquare $=28.9 \%$. Note1: Unstandardized coefficients were reported following by standard error in brackets. Note2: **p $<.01 ; * * \mathrm{p}<.001$

approximately two to three times lower than that reported in British (Groarke et al., 2020; Li \& Wang, 2020) and Japanese (Stickley et al., 2020) adult samples. There are two possible explanations for these differences. First, there are cross-regional and cultural variations in loneliness and social isolation, as reported by D'Hombres et al. (2018), based on Joint Research Centre reports across European countries. Second, there are methodological issues, in that some studies used a single item to measure loneliness (e.g., Li \& Wang, 2020). Nevertheless, our finding demonstrates the robustness of loneliness as a predictor of poor mental health and behavioural problems, including anxiety, depressive symptoms, and insomnia. This is consistent with previous reports of a causal relationship between loneliness and physical and mental health problems (Beutel et al., 2017; Hawkley \& Cacioppo, 2010; Lara et al., 2020; Leigh-Hunt et al., 2017; Holt-Lunstad et al., 2015; Lim et al., 2020; Solmi et al., 2020). Our results also reinforce a growing body of research showing a significant association between loneliness during the pandemic and greater anxiety and depressive symptoms (Killgore et al., 2020a; Okruszek et al., 2020; Palgi et al., 2020; Tso \& Park, 2020) and sleep problems (Grossman et al., 2021; Kokou-Kpolou et al., 2020a).

Furthermore, although a number of studies ( $\mathrm{Bu}$ et al., 2020b; Buecker et al., 2020; Groarke et al., 2020; Hawkley et al., 2020; Solmi et al., 2020; Wickens et al., 2021) have found that high levels of loneliness during the ongoing pandemic vary according to sex, age, marital status, place of

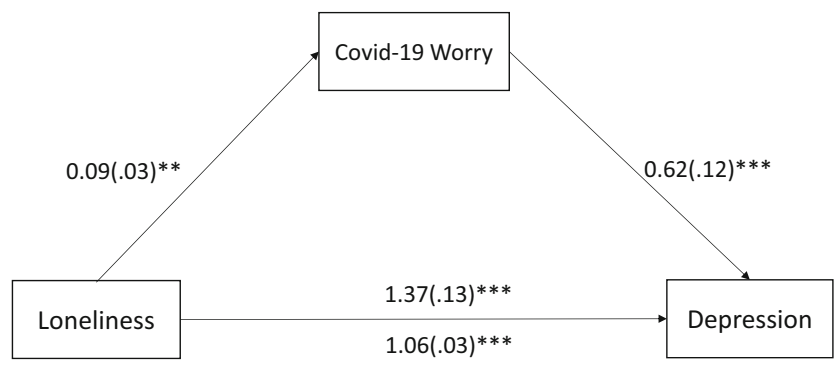

Fig. 6 Hypothesized path model showing mediated relationships between loneliness and depression through Covid-19 related worry. Rsquare $=33.7 \%$. Note1: Unstandardized coefficients were reported following by standard error in brackets. Note2: **p $<.01 ; * * \mathrm{p}<.001$

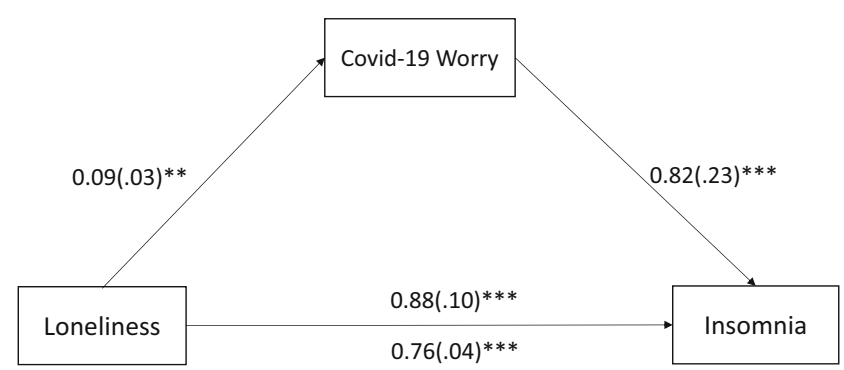

Fig. 7 Hypothesized path model showing mediated relationships between loneliness and insomnia through Covid-19 related worry. Rsquare $=15.0 \%$. Note 1 : Unstandardized coefficients were reported following by standard error in brackets. Note2: **p $<.01 ; * * \mathrm{p}<.001$

residence, and parenthood, we did not find that these variations predicted the severity of mental health and behavioural symptoms. Nevertheless, our findings showed that employment and working arrangements had a moderating effect on the relationship between loneliness and anxiety. Lonely feelings among individuals who had lost their job because of the pandemic were predictive of a severe risk of anxiety. In addition, individuals working from home who felt lonely tended to report more sleep problems. These results reflect the socioeconomic consequences of the pandemic on psychosocial wellbeing. The COVID-19 crisis has affected many business sectors, and tens of millions of individuals have lost or at risk to lose their jobs across the world. These socioeconomic consequences are found to translate in different forms of psychological distress including intolerance of uncertainty, anxiety, and social identity disturbance (Godinic et al., 2019).

Our findings also showed that preexisting health problems amplified the relationship between loneliness and insomnia. A large number of studies on COVID-19 have shown, albeit separately, that people with preexisting health problems report greater prevalence of loneliness (Bu et al., 2020b; Groarke et al., 2020), and experience poor mental health and behavioural problems (for reviews, see Wang et al., 2020; Xiong et al., 2020). Our findings reinforce these data and shed light on how the interaction between loneliness and preexisting physical or mental conditions exacerbates the risk of insomnia. It is somewhat surprising that this interaction was not associated with a higher likelihood of current anxiety and depressive symptoms. This may partially be explained by the shared mechanisms between previous medical and psychological conditions and current mental health status exacerbated by the COVID-19 crisis.

The current study expands the literature by suggesting that worrying about the COVID-19 crisis has both moderating and mediating effects on pathways from loneliness to anxiety, depressive symptoms, and insomnia. This pattern of findings is consistent with a study by Grossman et al. (2021), who found that sleep problems were associated with the loneliness - worry interaction during the pandemic. It also supports new studies of the mediating effects of worry on the relationships 
between different forms of loneliness (Anyan et al., 2020), perceived vulnerability to COVID-19 (Boyraz et al., 2020), and psychological distress, including anxiety, depressive symptoms, and posttraumatic stress.

\section{Implications}

Regarding the practical implications of the present study, our findings support the urgent need to pay attention to individuals with pre-existing health conditions The pandemic crisis with its consequent increase in fear, anxiety, etc., may have exacerbated their vulnerabilities and put them in heightened fragility (Megalakaki et al., 2021). More so, the overemphasis on the pandemic may have translated into less attention to other diseases thereby leaving this categories of persons with less access to health care. A particular attention should be also paid to those who have lost their job because of the pandemic. These individuals have a greater risk of becoming disconnected from the professional and social networks that previously protected them. They are vulnerable to concerns and negative repetitive thoughts about their future prospects, which may increase their anxiety. Even though governments in many Western countries have implemented programmes to assist those who have lost their job because of the SARS-CoV-2 pandemic, together with other socioeconomically disadvantaged people, these may prove inadequate over the long term, in terms of meeting their basic psychosocial needs (e.g., self-utility, self-accomplishment) (Cénat et al., 2020). Hence, these programmes should be accompanied by targeted mental health interventions. Attention should also be also paid to those who work from home, as abrupt transitions in work organization can lead to family and personal upheavals, which can affect sleep habits and homeostasis.

Furthermore, our results on the dual moderating and mediating effects of COVID-19-related worry indicate that interventions aimed at slowing down perseverative cognition linked to the COVID-19 crisis and reducing the negative valence of its content would help people feeling lonely to improve their mental health and wellbeing. Loneliness is a modifiable factor, such that combined interventions targeting both loneliness and worry could lead to positive health outcomes for at-risk individuals as the pandemic continues to make itself felt.

\section{Strengths and Limitations}

This represents the first study to examine the main effect of loneliness on mental health and behavioural outcomes induced by the COVID-19 crisis, as well as the moderating and mediating effects of biopsychosocial variables on these relationships. However, there were some limitations to the present study's findings regarding the extent to which they can be generalized. First, the self-reported nature of the study may have some peculiar responder biases which were not controlled for. Second, the cross-sectional nature of the data, coupled with the nonprobability sampling strategy we used, prevented us from establishing causal relationships between variables. A third limitation is related to the use of a single item to assess COVID-19-related worry. This may have promoted under-reporting of feelings of loneliness, which may disproportionately affect certain groups.

\section{Conclusion, Recommendation, and Future Direction}

In sum, the present findings indicate that loneliness was a major mental health concern during the first COVID-19 lockdown in France. It was associated with three mental health and behavioural outcomes, namely anxiety, depressive symptoms, and insomnia. These health consequences of loneliness varied across sociodemographic groups. Employment and working arrangements, living conditions, preexisting medical or psychological problems, and COVID-19 related worry were found to moderate relationships between loneliness and the three mental health and behavioural outcomes. Both moderating and mediating effects were observed for COVID-19-related worry. This robust evidence-based knowledge could inform treatment recommendations for tackling prolonged self-isolation and loneliness during the SARS-CoV-2 pandemic for specific vulnerable groups. Future investigations could be extended to other vulnerable groups such as individuals who lost their loved ones during the pandemic and may be of particular concern for increased feelings of loneliness (Kokou-Kpolou et al., 2020b). We encourage future research, if possible with longitudinal design, to use multiple-item scales in order to assess the multidimensional facets of loneliness (Anyan et al., 2020). Given that our mediation models suggested partial mediation, future research could build on the current study's findings by investigating other cognitive vulnerability mechanisms that may account for stronger relationships between feelings of loneliness and mental health and behavioural outcomes. Furthermore, for more in-depth understanding of these mechanisms, quantitative methods may be complemented with qualitative methods such as online photovoice which is a contextually appropriate method to exploring biopsychosocial well-being and concerns in the context of disasters (e.g., COVID-19 pandemic) (Tanhan, 2020; Tanhan \& Strack, 2020).

Supplementary Information The online version contains supplementary material available at https://doi.org/10.1007/s12144-021-02246-w.

Code Availability Not applicable.

Authors' Contributions Conceptualization: CKKK, OM; Methodology, Software and Formal analysis: CKKK; Validation: OM; Investigation: OM, CKKK; Data Curation: CKKK; Writing - Original Draft Preparation: CKKK; Writing - Review \& Editing: OM; Supervision: OM. 
Funding This article has been supported by the European center for humanities and social sciences (MESHS-Lille, France) and by the Regional Council Hauts-de-France.

Data Availability The datasets generated during and/or analysed during the current study are available from the corresponding author on reasonable request.

\section{Declarations}

Conflict of Interest The authors declare that they have no conflict of interest.

Ethics Approval The study protocol adhered to the guidelines of the Declaration of Helsinki and its later revisions, and was approved by the Ethics Committee of the Faculty of Psychotherapy and the Faculty of Psychology, Sigmund Freud University-Paris (LBP2CFYKAO1BEX87902).

Consent to Participate Informed consent was obtained from all individual participants for whom identifying information is included in this article.

Consent for Publication This manuscript has not been published elsewhere and is not under consideration by another journal. All authors are aware of its content and approve its submission and give their consent for publication in Current Psychology.

\section{References}

Anyan, F., Morote, R., \& Hjemdal, O. (2020). Prospective relations between loneliness in different relationships, metacognitive beliefs, worry and common mental health problems. Mental Health \& Prevention, 19, 200186. https://doi.org/10.1016/j.mhp.2020. 200186

Bastien, C. (2001). Validation of the insomnia severity index as an outcome measure for insomnia research. Sleep Medicine, 2(4), 297 307. https://doi.org/10.1016/S1389-9457(00)00065-4

Beam, C. R., \& Kim, A. J. (2020). Psychological sequelae of social isolation and loneliness might be a larger problem in young adults than older adults. Psychological Trauma: Theory, Research, Practice, and Policy, 12(S1), S58-S60. https://doi.org/10.1037/ tra0000774

Beutel, M. E., Klein, E. M., Brähler, E., Reiner, I., Jünger, C., Michal, M., Wiltink, J., Wild, P. S., Münzel, T., Lackner, K. J., \& Tibubos, A. N. (2017). Loneliness in the general population: Prevalence, determinants and relations to mental health. BMC Psychiatry, 17(1), 97. https://doi.org/10.1186/s12888-017-1262-X

Blais, F. C., Gendron, L., Mimeault, V., \& Morin, C. M. (1997). Assessment of insomnia: Validation of three questionnaires. L'Encéphale.

Borkovec, T. D., Robinson, E., Pruzinsky, T., \& DePree, J. A. (1983). Preliminary exploration of worry: Some characteristics and processes. Behaviour Research and Therapy, 21(1), 9-16. https://doi.org/ 10.1016/0005-7967(83)90121-3

Boyraz, G., Legros, D. N., \& Tigershtrom, A. (2020). COVID-19 and traumatic stress: The role of perceived vulnerability, COVID-19related worries, and social isolation. Journal of Anxiety Disorders, 76, 102307. https://doi.org/10.1016/j.janxdis.2020.102307

Brooks, S. K., Webster, R. K., Smith, L. E., Woodland, L., Wessely, S., Greenberg, N., \& Rubin, G. J. (2020). The psychological impact of quarantine and how to reduce it: Rapid review of the evidence. The
Lancet, 395(10227), 912-920. https://doi.org/10.1016/S0140-6736 (20)30460-8

Brosschot, J. F., Gerin, W., \& Thayer, J. F. (2006). The perseverative cognition hypothesis: A review of worry, prolonged stress-related physiological activation, and health. Journal of Psychosomatic Research, 60(2), 113-124. https://doi.org/10.1016/j.jpsychores. 2005.06.074

Bu, F., Steptoe, A., \& Fancourt, D. (2020a). Loneliness during a strict lockdown: Trajectories and predictors during the COVID-19 pandemic in 38,217 United Kingdom adults. Social Science \& Medicine, 265, 113521. https://doi.org/10.1016/j.socscimed.2020. 113521

Bu, F., Steptoe, A., \& Fancourt, D. (2020b). Who is lonely in lockdown? Cross-cohort analyses of predictors of loneliness before and during the COVID-19 pandemic. Public Health, 186, 31-34. https://doi. org/10.1016/j.puhe.2020.06.036

Buecker, S., Horstmann, K. T., Krasko, J., Kritzler, S., Terwiel, S., Kaiser, T., \& Luhmann, M. (2020). Changes in daily loneliness for German residents during the first four weeks of the COVID-19 pandemic. Social Science \& Medicine, 265, 113541. https://doi.org/ 10.1016/j.socscimed.2020.113541

Cénat, J. M., Dalexis, R. D., Kokou-Kpolou, C. K., Mukunzi, J. N., \& Rousseau, C. (2020). Social inequalities and collateral damages of the COVID-19 pandemic: When basic needs challenge mental health care. International Journal of Public Health, 65(6), 717718. https://doi.org/10.1007/s00038-020-01426-y

Cénat, J. M., Blais-Rochette, C., Kokou-Kpolou, C. K., Noorishad, P.-G., Mukunzi, J. N., McIntee, S.-E., Dalexis, R. D., Goulet, M.-A., \& Labelle, P. R. (2021). Prevalence of symptoms of depression, anxiety, insomnia, posttraumatic stress disorder, and psychological distress among populations affected by the COVID-19 pandemic: A systematic review and meta-analysis. Psychiatry Research, 295, 113599. https://doi.org/10.1016/j.psychres.2020.113599

Clancy, F., Prestwich, A., Caperon, L., Tsipa, A., \& O’Connor, D. B. (2020). The association between worry and rumination with sleep in non-clinical populations: A systematic review and meta-analysis. Health Psychology Review, 14(4), 427-448. https://doi.org/10. 1080/17437199.2019.1700819

Cochran, W. (1977). Sampling Techniques (3rd Ed.). New York: John Wiley and Sons, Inc.

Cohen, P., Cohen, P., West, S. G., \& Aiken, L. S. (2014). Applied multiple regression/correlation analysis for the behavioral sciences. https://doi.org/10.4324/9781410606266.

D'Hombres, B., Sylke, S., Barjaková, M., \& Mendonca, F. T. (2018). Loneliness-an unequally shared burden in Europe. Technical report. https://doi.org/10.13140/RG.2.2.21745.33128

Fritz, M. S., \& MacKinnon, D. P. (2007). Required sample size to detect the mediated effect. Psychological Science, 18(3), 233-239. https:// doi.org/10.1111/J.1467-9280.2007.01882.X

Godinic, D., Obrenovic, B., \& Khudaykulov, A. (2019). Effects of economic uncertainty on mental health in the COVID-19 pandemic context: Social identity disturbance, job uncertainty and psychological well-being model. International journal of innovation and economics development, 6, 61-74. https://doi.org/10.18775/ijied.18497551-7020.2015.61.2005

Groarke, J. M., Berry, E., Graham-Wisener, L., McKenna-Plumley, P. E., McGlinchey, E., \& Armour, C. (2020). Loneliness in the UK during the COVID-19 pandemic: Cross-sectional results from the COVID19 psychological wellbeing study. PLoS One, 15(9), e0239698. https://doi.org/10.1371/journal.pone.0239698

Grossman, E. S., Hoffman, Y. S. G., Palgi, Y., \& Shrira, A. (2021). COVID-19 related loneliness and sleep problems in older adults: Worries and resilience as potential moderators. Personality and Individual Differences, 168, 110371. https://doi.org/10.1016/j.paid. 2020.110371 
Hawkley, L. C., Buecker, S., Kaiser, T., \& Luhmann, M. (2020). Loneliness from young adulthood to old age: Explaining age differences in loneliness. International journal of behavioral development, 016502542097104. https://doi.org/10.1177/ $0165025420971048,016502542097104$.

Hawkley, L. C., \& Cacioppo, J. T. (2010). Loneliness matters: A theoretical and empirical review of consequences and mechanisms. Annals of Behavioral Medicine, 40(2), 218-227. https://doi.org/10. 1007/s12160-010-9210-8

Holt-Lunstad, J., Smith, T. B., Baker, M., Harris, T., \& Stephenson, D. (2015). Loneliness and social isolation as risk factors for mortality. Perspectives on Psychological Science, 10(2), 227-237. https://doi. org/10.1177/1745691614568352

Hughes, M. E., Waite, L. J., Hawkley, L. C., \& Cacioppo, J. T. (2004). A short scale for measuring loneliness in large surveys. Research on Aging, 26(6), 655-672. https://doi.org/10.1177/0164027504268574

Jeste, D. V., Lee, E. E., \& Cacioppo, S. (2020). Battling the modern behavioral epidemic of loneliness. JAMA Psychiatry, 77(6), 553554. https://doi.org/10.1001/jamapsychiatry.2020.0027

Kämpfen, F., Kohler, I. v., Ciancio, A., Bruine de Bruin, W., Maurer, J., \& Kohler, H.-P. (2020). Predictors of mental health during the Covid-19 pandemic in the US: Role of economic concerns, health worries and social distancing. PLoS One, 15(11), e0241895. https:// doi.org/10.1371/journal.pone.0241895

Kennedy-Shaffer, L. (2019). Before $p<0.05$ to beyond $p<0.05$ : Using history to contextualize $\mathrm{p}$-values and significance testing. https://doi. org/10.1080/00031305.2018.1537891, 73(sup1), 82-90. https://doi. org/10.1080/00031305.2018.1537891.

Killgore, W. D. S., Cloonan, S. A., Taylor, E. C., \& Dailey, N. S. (2020a). Loneliness: A signature mental health concern in the era of COVID19. Psychiatry Research, 290, 113117. https://doi.org/10.1016/j. psychres.2020.113117

Killgore, W. D. S., Cloonan, S. A., Taylor, E. C., Miller, M. A., \& Dailey, N. S. (2020b). Three months of loneliness during the COVID-19 lockdown. Psychiatry Research, 293, 113392. https://doi.org/10. 1016/j.psychres.2020.113392

Killgore, W. D. S., Cloonan, S. A., Taylor, E. C., Lucas, D. A., \& Dailey, N. S. (2020c). Loneliness during the first half-year of COVID-19 lockdowns. Psychiatry Research, 294, 113551. https://doi.org/10. 1016/j.psychres.2020.113551

Kircanski, K., Thompson, R. J., Sorenson, J. E., Sherdell, L., \& Gotlib, I. H. (2015). Rumination and worry in daily life. Clinical Psychological Science, 3(6), 926-939. https://doi.org/10.1177/ 2167702614566603

Kokou-Kpolou, C. K., Megalakaki, O., Laimou, D., \& Kousouri, M. (2020b). Insomnia during COVID-19 pandemic and lockdown: Prevalence, severity, and associated risk factors in French population. Psychiatry Research, 290, 113128. https://doi.org/10.1016/j. psychres.2020.113128

Kokou-Kpolou, C. K., Fernández-Alcántara, M., \& Cénat, J. M. (2020a). Prolonged grief related to COVID-19 deaths: Do we have to fear a steep rise in traumatic and disenfranchised griefs? Psychological trauma: Theory, research, practice, and policy? 12(S1), S94-S95. DOI, 12, S94-S95. https://doi.org/10.1037/tra0000798

Kroeke, K., \& Spitzer, R. L. (2002). The PHQ-9: A new depression diagnostic and severity measure. Psychiatric Annals, 32(9), 509515. https://doi.org/10.3928/0048-5713-20020901-06

Lara, E., Moreno-Agostino, D., Martín-María, N., Miret, M., Rico-Uribe, L. A., Olaya, B., Cabello, M., Haro, J. M., \& Ayuso-Mateos, J. L. (2020). Exploring the effect of loneliness on all-cause mortality: Are there differences between older adults and younger and middle-aged adults? Social Science \& Medicine, 258, 113087. https://doi.org/10. 1016/j.socscimed.2020.113087

Lee, C. M., Cadigan, J. M., \& Rhew, I. C. (2020). Increases in loneliness among young adults during the COVID-19 pandemic and association with increases in mental health problems. Journal of Adolescent
Health, 67(5), 714-717. https://doi.org/10.1016/j.jadohealth.2020. 08.009

Leigh-Hunt, N., Bagguley, D., Bash, K., Turner, V., Turnbull, S., Valtorta, N., \& Caan, W. (2017). An overview of systematic reviews on the public health consequences of social isolation and loneliness. Public Health, 152, 157-171. https://doi.org/10.1016/j.puhe.2017. 07.035

Li, L. Z., \& Wang, S. (2020). Prevalence and predictors of general psychiatric disorders and loneliness during COVID-19 in the United Kingdom. Psychiatry Research, 291, 113267. https://doi.org/10. 1016/j.psychres.2020.113267

Lim, M. H., Holt-Lunstad, J., \& Badcock, J. C. (2020). Loneliness: Contemporary insights into causes, correlates, and consequences. Social Psychiatry and Psychiatric Epidemiology, 55(7), 789-791. https://doi.org/10.1007/s00127-020-01891-z

Luchetti, M., Lee, J. H., Aschwanden, D., Sesker, A., Strickhouser, J. E., Terracciano, A., \& Sutin, A. R. (2020). The trajectory of loneliness in response to COVID-19. American Psychologist, 75(7), 897-908. https://doi.org/10.1037/amp0000690

Marvaldi, M., Mallet, J., Dubertret, C., Moro, M. R., \& Guessoum, S. B. (2021). Anxiety, depression, trauma-related, and sleep disorders among healthcare workers during the COVID-19 pandemic: A systematic review and meta-analysis. Neuroscience \& Biobehavioral Reviews, 126, 252-264. https://doi.org/10.1016/J.NEUBIOREV. 2021.03.024

McGinty, E. E., Presskreischer, R., Han, H., \& Barry, C. L. (2020). Psychological distress and loneliness reported by US adults in 2018 and April 2020. JAMA, 324(1), 93. https://doi.org/10.1001/ jama.2020.9740, 324, 94.

Megalakaki, O., Kokou-Kpolou, C. K., Vaudé, J., Park, S., Iorfa, S. K., Cénat, J. M., \& Derivois, D. (2021). Does peritraumatic distress predict PTSD, depression and anxiety symptoms during and after COVID-19 lockdown in France? A prospective longitudinal study. Journal of Psychiatric Research, 137, 81-88. https://doi.org/10. 1016/j.jpsychires.2021.02.035

Morin, C. M., Belleville, G., Bélanger, L., \& Ivers, H. (2011). The insomnia severity index: Psychometric indicators to detect insomnia cases and evaluate treatment response. Sleep, 34(5), 601-608. https://doi.org/10.1093/sleep/34.5.601

Muthen, L. K., \& Muthen, B. O. (2017). Statistical analysis with latent variables User's guide (8th edition). Los Angeles, CA : Authors.

Okruszek, Ł., Aniszewska-Stańczuk, A., Piejka, A., Wiśniewska, M., \& Żurek, K. (2020). Safe but lonely? Loneliness, anxiety, and depression symptoms and COVID-19. Frontiers in Psychology, 11. https:// doi.org/10.3389/fpsyg.2020.579181

Palgi, Y., Shrira, A., Ring, L., Bodner, E., Avidor, S., Bergman, Y., Cohen-Fridel, S., Keisari, S., \& Hoffman, Y. (2020). The loneliness pandemic: Loneliness and other concomitants of depression, anxiety and their comorbidity during the COVID-19 outbreak. Journal of Affective Disorders, 275, 109-111. https://doi.org/10.1016/j.jad. 2020.06 .036

Paluszek, M. M., Asmundson, A. J. N., Landry, C. A., McKay, D., Taylor, S., \& Asmundson, G. J. G. (2021). Effects of anxiety sensitivity, disgust, and intolerance of uncertainty on the COVID stress syndrome: A longitudinal assessment of transdiagnostic constructs and the behavioural immune system. https://doi.org/10.1080/ 16506073.2021.1877339, 50(3), 191-203.

Peng, S., Yang, X. Y., Yang, T., Zhang, W., \& Cottrell, R. R. (2021). Uncertainty stress, and its impact on disease fear and prevention behavior during the COVID-19 epidemic in China: A panel study. American Journal of Health Behavior, 45(4), 334-341. https://doi. org/10.5993/AJHB.45.2.12

Preacher, K.J., Hayes, A.F. (2008). Asymptotic and resampling strategies for assessing and comparing indirect effects in multiple mediator models. Behavior Research Methods, 40, 879-891. https://doi.org/ 10.3758/BRM.40.3.879 
Rosenberg, M., Luetke, M., Hensel, D., Kianersi, S., Fu, T., \& Herbenick, D. (2021). Depression and loneliness during April 2020 COVID-19 restrictions in the United States, and their associations with frequency of social and sexual connections. Social Psychiatry and Psychiatric Epidemiology., 56, 1221-1232. https://doi.org/10. 1007/s00127-020-02002-8

Saltzman, L. Y., Hansel, T. C., \& Bordnick, P. S. (2020). Loneliness, isolation, and social support factors in post-COVID-19 mental health. Psychological Trauma: Theory, Research, Practice, and Policy, 12(S1), S55-S57. https://doi.org/10.1037/tra0000703

Solmi, M., Veronese, N., Galvano, D., Favaro, A., Ostinelli, E. G., Noventa, V., Favaretto, E., Tudor, F., Finessi, M., Shin, J. I., Smith, L., Koyanagi, A., Cester, A., Bolzetta, F., Cotroneo, A., Maggi, S., Demurtas, J., De Leo, D., \& Trabucchi, M. (2020). Factors associated with loneliness: An umbrella review of observational studies. Journal of Affective Disorders, 271, 131-138. https:// doi.org/10.1016/j.jad.2020.03.075

Spitzer, R. L., Kroenke, K., Williams, J. B. W., \& Löwe, B. (2006). A brief measure for assessing generalized anxiety disorder. Archives of Internal Medicine, 166(10), 1092. https://doi.org/10.1001/archinte. 166.10.1092

Stickley, A., Matsubayashi, T., \& Ueda, M. (2020). Loneliness and COVID-19 preventive behaviours among Japanese adults. Journal of Public Health., 43, 53-60. https://doi.org/10.1093/pubmed/ fdaa151

Tanhan, A. (2020). Utilizing online Photovoice (OPV) methodology to address biopsychosocial spiritual economic issues and wellbeing during COVID-19: Adapting OPV to Turkish. Turkish Studies, 15(4), 1029-1086. https://doi.org/10.7827/TurkishStudies.44451

Tanhan, A., \& Strack, R. W. (2020). Online photovoice to explore and advocate for Muslim biopsychosocial spiritual wellbeing and issues: Ecological systems theory and ally development. Current Psychology, 39(6), 2010-2025. https://doi.org/10.1007/S12144020-00692-6

Tousignant, O. H., Taylor, N. D., Suvak, M. K., \& Fireman, G. D. (2019). Effects of rumination and worry on sleep. Behavior Therapy, 50(3), 558-570. https://doi.org/10.1016/j.beth.2018.09.005
Tso, I. F., \& Park, S. (2020). Alarming levels of psychiatric symptoms and the role of loneliness during the COVID-19 epidemic: A case study of Hong Kong. Psychiatry Research, 293, 113423. https://doi. org/10.1016/j.psychres.2020.113423

van der Velden, P. G., Contino, C., Das, M., van Loon, P., \& Bosmans, M. W. G. (2020). Anxiety and depression symptoms, and lack of emotional support among the general population before and during the COVID-19 pandemic. A prospective national study on prevalence and risk factors. Journal of Affective Disorders, 277, 540-548. https://doi.org/10.1016/j.jad.2020.08.026

van der Velden, P. G., Hyland, P., Contino, C., von Gaudecker, H.-M., Muffels, R., \& Das, M. (2021). Anxiety and depression symptoms, the recovery from symptoms, and loneliness before and after the COVID-19 outbreak among the general population: Findings from a Dutch population-based longitudinal study. PLoS One, 16(1), e0245057. https://doi.org/10.1371/journal.pone.0245057

Wang, Y., Kala, M. P., \& Jafar, T. H. (2020). Factors associated with psychological distress during the coronavirus disease 2019 (COVID-19) pandemic on the predominantly general population: A systematic review and meta-analysis. PLoS One, 15(12), e0244630. https://doi.org/10.1371/journal.pone.0244630

Wickens, C. M., McDonald, A. J., Elton-Marshall, T., Wells, S., Nigatu, Y. T., Jankowicz, D., \& Hamilton, H. A. (2021). Loneliness in the COVID-19 pandemic: Associations with age, gender and their interaction. Journal of Psychiatric Research, 136, 103-108. https:// doi.org/10.1016/j.jpsychires.2021.01.047

Xiong, J., Lipsitz, O., Nasri, F., Lui, L. M. W., Gill, H., Phan, L., ChenLi, D., Iacobucci, M., Ho, R., Majeed, A., \& McIntyre, R. S. (2020). Impact of COVID-19 pandemic on mental health in the general population: A systematic review. Journal of Affective Disorders, 277, 55-64. https://doi.org/10.1016/j.jad.2020.08.001

Publisher's Note Springer Nature remains neutral with regard to jurisdictional claims in published maps and institutional affiliations. 